\title{
The LHCb upgrade
}

\author{
Heinrich Schindler* on behalf of the LHCb collaboration \\ CERN \\ E-mail: heinrich.schindler@cern.ch
}

The upgrade of the LHCb experiment, with its installation scheduled for the second long shutdown (LS2) of the Large Hadron Collider (LHC), will transform the data acquisition and processing architecture to a fully software-based event selection in a CPU farm. This also requires a redesign of major subdetector components addressing the challenges posed by $40 \mathrm{MHz}$ readout, increased occupancy and radiation damage. The upgraded experiment is expected to reach statistical sensitivities comparable to the theoretical uncertainties and will broaden further its physics programme.

XV International Conference on Hadron Spectroscopy-Hadron 2013

4-8 November 2013

Nara, Japan

${ }^{*}$ Speaker. 

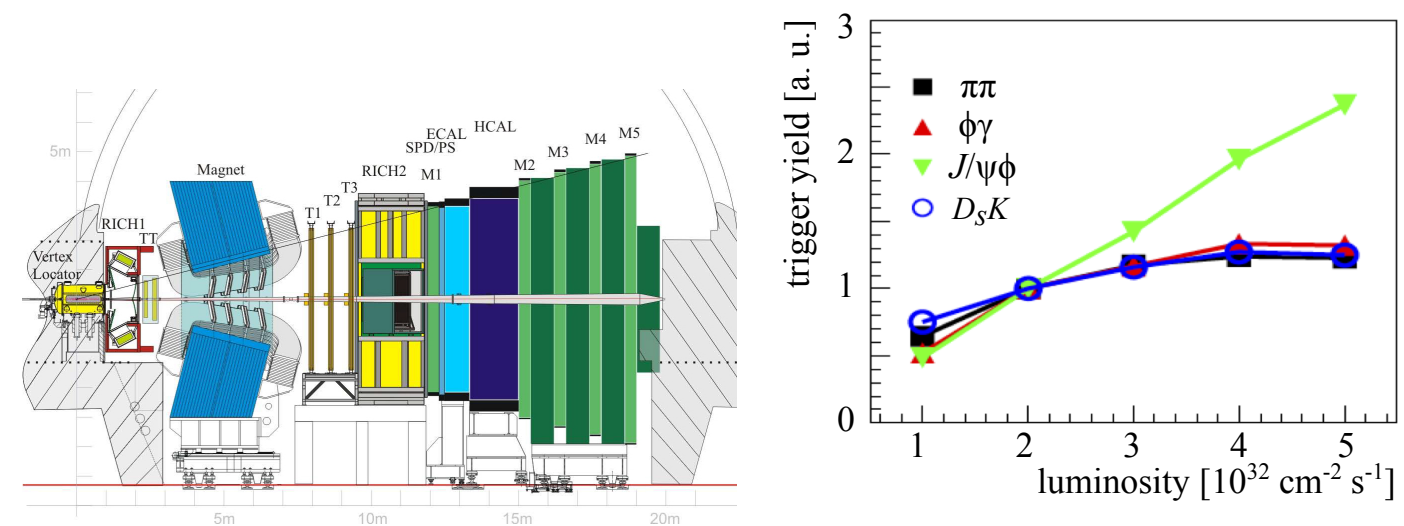

Figure 1: Left: layout of the existing LHCb detector. Right: simulated yield of the L0 trigger as function of luminosity for selected decays of $B$ mesons (reproduced from Ref. [1]). The yields of each channel are normalised with respect to the values at $\mathscr{L}=2 \times 10^{32} \mathrm{~cm}^{-2} \mathrm{~s}^{-1}$.

\section{Introduction}

$\mathrm{LHCb}$ is an LHC experiment designed for heavy quark flavour physics. The detector is a single-arm forward spectrometer, covering the pseudorapidity range $2<\eta<5$. The tracking system consists of an array of silicon sensors surrounding the interaction point, known as the Vertex Locator (VELO), followed by one tracking station upstream and three tracking stations downstream of a $4 \mathrm{Tm}$ dipole magnet. Particle identification is provided by two ring-imaging Cherenkov (RICH) detectors, a system of calorimeters and five stations of muon chambers (Fig. 1 left). Calorimeter and muon systems provide $40 \mathrm{MHz}$ input to the hardware Level-0 (L0) trigger, which based on transverse momentum and energy cuts reduces the event rate to the $1 \mathrm{MHz}$ readout rate of the other subdetectors. The L0 trigger is followed by a software-based high-level trigger (HLT) which performs a full event reconstruction.

During Run 1 of the LHC, the experiment has shown excellent performance and has proven to be robust, operating in 2012 at an instantaneous (levelled) luminosity of $\mathscr{L}=4 \times 10^{32} \mathrm{~cm}^{-2} \mathrm{~s}^{-1}$ (twice the design luminosity). Key performance figures of merit include a momentum resolution of $0.35 \%-0.55 \%$, a decay time resolution of $\lesssim 50 \mathrm{fs}$, a $\sim 95 \%$ kaon identification efficiency (for $\sim 5 \% \pi \rightarrow K$ mis-identification), and an energy resolution in the electromagnetic calorimeter of $\sigma_{E} / E \sim 10 \% / \sqrt{E[\mathrm{GeV}]} \oplus 1 \%$.

\section{Upgrade strategy and physics prospects}

During Run 1 (2010 - 2012), LHCb has collected a sample of $3 \mathrm{fb}^{-1}$. Data taking will be resumed in 2015, and during LHC Run 2 the experiment is expected to record about $5 \mathrm{fb}^{-1}$. After the second long shutdown (LS2), scheduled for 2018/19, the data taking time necessary for doubling the statistics would become too long to be worthwhile. A significant increase in instantaneous luminosity is therefore required.

The main "bottleneck" which prevents running at higher luminosity with the existing detector is the $1 \mathrm{MHz}$ readout rate in combination with the limited discriminating power of the L0 hardware 
trigger. The L0 efficiency is significantly higher for muon-triggered decays than for purely hadronic channels. In case of the latter, a tight cut on the transverse energy of several $\mathrm{GeV}$ needs to be applied to keep the output rate below $1 \mathrm{MHz}$. As shown in Fig. 1 (right), this leads to a saturation of the signal yield for these channels and thus limits the maximum useable luminosity to approximately $4 \times 10^{32} \mathrm{~cm}^{-2} \mathrm{~s}^{-1}$.

In the upgraded experiment, this limitation will be overcome by reading out the whole detector at every bunch-crossing and moving to a fully software-based ${ }^{1}$ trigger $[1,2]$. As a consequence, the detector front-end electronics need to be redesigned and - due to the tight integration of sensors and readout ASICs - the silicon detectors need to be replaced.

The target instantaneous luminosity is $\mathscr{L}=2 \times 10^{33} \mathrm{~cm}^{-2} \mathrm{~s}^{1}$, and the upgraded experiment is expected to collect $\sim 5 \mathrm{fb}^{-1}$ per year. With an integrated luminosity of $50 \mathrm{fb}^{-1}$, the statistical sensitivities are expected to become comparable to the theoretical uncertainties. A recent update of the expected sensitivities, extrapolated from existing measurements and taking into account improvements in the trigger yield due to the removal of the L0 stage, can be found in Ref. [3].

For several areas of flavour physics, for instance $B_{s}^{0}$ and $b$-hadron decays or CP violation and mixing in charm, the upgraded $\mathrm{LHCb}$ experiment will be in a unique position. A key channel for probing effects of new physics in the $B_{s}^{0}$ system, which is covered only by $\mathrm{LHCb}$, is e.g. $B_{s}^{0} \rightarrow$ $\phi \phi$. Already now, $\mathrm{LHCb}$ is also exploring topics outside the core programme which exploit the kinematic coverage of the experiment. These studies will be boosted further with the upgrade due to the enhanced flexibility of the trigger. The upgraded LHCb experiment can thus be considered a general-purpose experiment in the forward region.

\section{Tracking}

\subsection{Vertex Locator (VELO)}

The present Vertex Locator consists of 21 stations of $R$ and $\phi$ measuring microstrip sensors oriented perpendicularly to the beam line. The detector operates in vacuum and the two halves (left and right) can be moved into and out of the beam.

The upgraded VELO [4] will use hybrid silicon pixel detectors based on an ASIC, dubbed "Velopix", with a $256 \times 256$ matrix of $55 \times 55 \mu \mathrm{m}^{2}$ pixels. As its direct predecessor Timepix 3 , the Velopix ASIC will operate in data-driven readout mode, but at a significantly higher rate (900 MHits / s peak pixel hit rate). The cooling is based on microchannel technology with bi-phase $\mathrm{CO}_{2}$ as coolant. The ability of the microchannel plates to withstand high pressure (up to 700 bar) has been demonstrated and endurance testing has started. Primary (beam) and secondary (VELO) vacuum are separated by thin aluminium boxes with corrugated walls known as the "RF foils". The inner radius of the upgraded RF foil is foreseen to be reduced from its present value of $5.5 \mathrm{~mm}$ to $3.5 \mathrm{~mm}$. As a consequence, the sensitive area of the detector can also be brought closer to the beam, to a distance of $5.1 \mathrm{~mm}$ from the nominal beam line. This results in a significantly improved impact parameter resolution (Fig. 2 left) and, consequently, an improved decay time resolution [3] with respect to the existing detector. On the other hand, the pixels closest to the interaction point

\footnotetext{
${ }^{1}$ Initially, a low-level hardware trigger (similar to the existing L0 trigger) will be kept to throttle the input to the HLT depending on the size of CPU farm.
} 

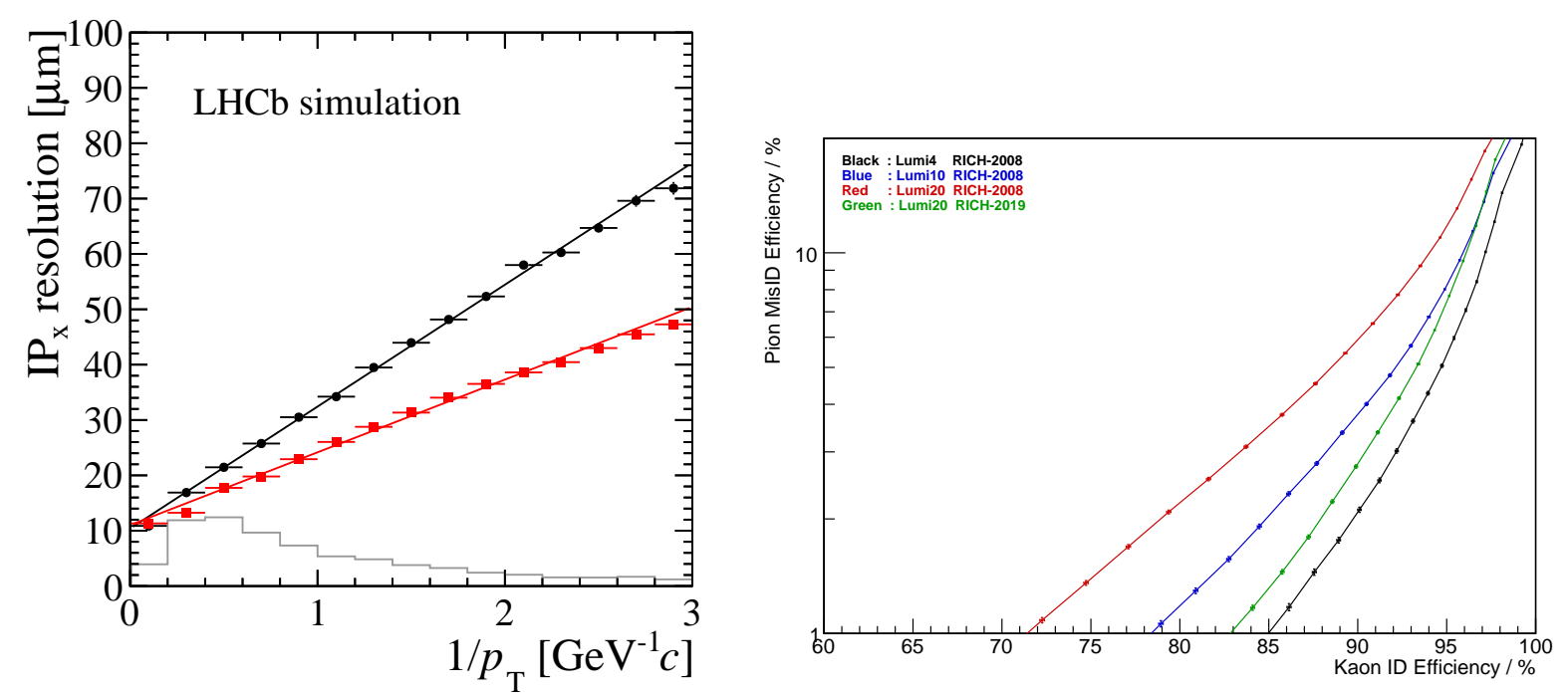

Figure 2: Left: resolution of the impact parameter $x$-component at upgrade beam conditions for existing VELO (black circles) and upgraded VELO (red squares), reproduced from Ref. [4]. Right: particle identification performance with the existing RICH1 layout at $\mathscr{L}=4 \times 10^{32} \mathrm{~cm}^{-2} \mathrm{~s}^{-1}$ (black), $1 \times 10^{33} \mathrm{~cm}^{-2} \mathrm{~s}^{-1}$ (blue) and $2 \times 10^{33} \mathrm{~cm}^{-2} \mathrm{~s}^{-1}$ and with the redesigned RICH1 at $2 \times 10^{33} \mathrm{~cm}^{-2} \mathrm{~s}^{-1}$ (green), reproduced from Ref. [5].

will accumulate a high fluence $\left(\sim 8 \times 10^{15} \mathrm{n}_{\mathrm{eq}} \mathrm{cm}^{-2}\right.$ after $\left.50 \mathrm{fb}^{-1}\right)$ and the "hottest" ASICs will need to cope with a high data rate $(\sim 15 \mathrm{Gbit} / \mathrm{s})$.

\subsection{Tracker Stations}

At present, the upstream tracker station (TT) and the inner regions (close to the beam pipe) of the downstream stations (IT) are silicon strip detectors, while the outer regions of the downstream stations are instrumented with straw tubes (OT). The main problem in the upgrade scenario is the high occupancy $(\sim 40 \%)$ of the straw tubes in the central region.

The baseline upgrade design foresees the replacement of IT and OT by a scintillating fibre tracker, covering the full area of the existing tracking stations $\left(6 \times 5 \mathrm{~m}^{2}\right)$. Each station comprises five staggered layers of $2.5 \mathrm{~m}$ long scintillating fibres with a diameter of $250 \mu \mathrm{m}$. The fibres are read out at the ends away from the beam by silicon photomultipliers (SiPMs) which are located outside the acceptance. The opposite fibre ends will be equipped with mirrors to maximise the amount of collected light. In order to keep the dark count rate due to the neutron fluence expected in the upgraded experiment at an acceptable level, the SiPMs need to be operated at a temperature of $\sim-40^{\circ} \mathrm{C}$.

The upstream tracking station will be instrumented with silicon strip sensors with finer granularity. This subdetector is expected to play an enhanced role in the upgrade trigger by providing a fast momentum estimate. 


\section{Particle Identification}

\subsection{RICH}

Due to high occupancy, the aerogel radiator in RICH1 will be removed, leaving the gaseous radiators $\mathrm{CF}_{4}$ in $\mathrm{RICH} 1$ and $\mathrm{C}_{4} \mathrm{~F}_{10}$ in $\mathrm{RICH} 2$. As a consequence of the $40 \mathrm{MHz}$ readout, all photon detectors need to be replaced. The R\&D is focussed on multi-anode photomultiplier tubes, a promising candidate is the R11265 MaPMT from Hamamatsu. Hybrid photon detectors (HPDs) with an external $40 \mathrm{MHz}$ readout ASIC are being considered as an alternative solution.

Simulations show that - without changes - the occupancy in RICH1 would become very high at the upgrade luminosity leading to a degradation in PID performance. The optics system of RICH1 has therefore been redesigned to increase the focal length and spread out the rings over more photon detectors. Figure 2 (right) shows that with these modifications, the PID performance at upgrade conditions is close to that of the existing RICH at the 2012 luminosity [5].

\subsection{TORCH}

In order to enhance the particle identification performance for low momentum tracks $(<$ $10 \mathrm{GeV} / c$ ), a novel detector concept, dubbed "TORCH", based on time-of-flight measurement has been proposed [6]. The required time resolution is $\sim 15$ ps per track, corresponding to $\sim 70 \mathrm{ps}$ resolution per photon. Micro-channel plates have been identified as potential photon detectors. The TORCH does not form part of the upgrade during LS2, but could be added at a later stage.

\subsection{Calorimeter and muon systems}

The first muon station (M1) as well as the preshower (PS) and scintillating pad detector (SPD) will be removed due to their reduced role in the upgrade trigger scheme. The calorimeter frontend electronics will be redesigned to compensate for the reduced gain at which the PMTs will be operated by an increased gain in the electronics. A replacement of some of the inner modules of the electromagnetic calorimeter is foreseen for the long shutdown 3 (LS3). Additional shielding will be installed to reduce the occupancy in the muon stations and $\mathrm{R} \& \mathrm{D}$ on more rate-tolerant detector technologies is underway.

\section{References}

[1] LHCb collaboration, Letter of Intent for the LHCb Upgrade, CERN-LHCC-2011-001

[2] LHCb collaboration, Framework TDR for the LHCb Upgrade, CERN-LHCC-2012-007

[3] LHCb collaboration, Updated sensitivity projections for the LHCb Upgrade, LHCb-PUB-2013-015

[4] LHCb collaboration, LHCb VELO Upgrade Technical Design Report, CERN-LHCC-2013-021

[5] LHCb collaboration, LHCb PID Upgrade Technical Design Report, CERN-LHCC-2013-022

[6] M. Charles and R. Forty, TORCH: Time of Flight Identification with Cherenkov Radiation, Nucl. Instr. Meth. A 639 (2011), 173 